\title{
Altered Age-Related Trajectories of Amygdala-Prefrontal Circuitry in Adolescents at Clinical High Risk for Psychosis: A Preliminary Study
}

\author{
Dylan G. Gee ${ }^{\mathrm{a}}$, Katherine H. Karlsgodt ${ }^{\mathrm{a}}$, Carrie E. Bearden ${ }^{\mathrm{a}}$, Matthew D. Lieberman ${ }^{\mathrm{a}}$, \\ Aysenil Belger $^{\mathrm{b}}$, Diana O. Perkins ${ }^{\mathrm{b}}$, Doreen M. Olvet ${ }^{\mathrm{c}}$, Barbara A. Cornblatt ${ }^{\mathrm{c}}$, Todd \\ Constable $^{d}$, Scott W. Woods ${ }^{d}$, Jean Addington ${ }^{e}$, Kristin S. Cadenhead ${ }^{f}$, Thomas H. \\ McGlashan $^{d}$, Larry J. Seidman ${ }^{g}$, Ming T. Tsuang ${ }^{f}$, Elaine F. Walker ${ }^{h}$, and Tyrone D. Cannon ${ }^{a}$ \\ on behalf of the NAPLS Consortium \\ aUniversity of California, Los Angeles, Departments of Psychology and Psychiatry, Los Angeles, \\ CA, USA \\ bUniversity of North Carolina, Chapel Hill, Department of Psychiatry, Chapel Hill, NC, USA \\ 'Zucker Hillside Hospital, Department of Psychiatry Research, Glen Oaks, NY, USA \\ dYale University, Department of Psychiatry, New Haven, CT, USA \\ eUniversity of Calgary, Department of Psychiatry, Calgary, Alberta, Canada \\ fUniversity of California, San Diego, Department of Psychiatry, La Jolla, CA, USA \\ gBeth Israel Deaconess Medical Center, Department of Psychiatry, Boston, MA, USA \\ hEmory University, Department of Psychology, Atlanta, GA, USA
}

\section{Abstract}

Emotion processing deficits are prominent in schizophrenia and exist prior to the onset of overt psychosis. However, developmental trajectories of neural circuitry subserving emotion regulation and the role that they may play in illness onset have not yet been examined in patients at risk for psychosis. The present study employed a cross-sectional analysis to examine age-related functional activation in amygdala and prefrontal cortex, as well as functional connectivity between these regions, in adolescents at clinical high risk (CHR) for psychosis relative to typically developing adolescents. Participants $(n=34)$ performed an emotion processing fMRI task, including emotion labeling, emotion matching, and non-emotional control conditions. Regression analyses were used to predict activation in the amygdala and ventrolateral prefrontal cortex (vlPFC) based on age, group, sex, and the interaction of age by group. CHR adolescents exhibited altered age-related variation in amygdala and vlPFC activation, relative to controls. Controls displayed decreased amygdala and increased vlPFC activation with age, while patients exhibited the opposite pattern (increased amygdala and decreased vlPFC activation), suggesting a failure of prefrontal cortex to regulate amygdala reactivity. Moreover, a psychophysiological interaction

(C) 2011 Elsevier B.V. All rights reserved.

Corresponding author: Tyrone D. Cannon, Ph.D., University of California, Los Angeles, 1285 Franz Hall, Los Angeles, CA 90095-1563, Phone: (310) 794-9673, Fax: (310) 794-9740, cannon@psych.ucla.edu.

* A complete list of study personnel involved in the NAPLS Consortium appears in the Appendix

Publisher's Disclaimer: This is a PDF file of an unedited manuscript that has been accepted for publication. As a service to our customers we are providing this early version of the manuscript. The manuscript will undergo copyediting, typesetting, and review of the resulting proof before it is published in its final citable form. Please note that during the production process errors may be discovered which could affect the content, and all legal disclaimers that apply to the journal pertain. 
analysis revealed decreased amygdala-prefrontal functional connectivity among CHR adolescents, consistent with disrupted brain connectivity as a vulnerability factor in schizophrenia. These results suggest that the at-risk syndrome is marked by abnormal development and functional connectivity of neural systems subserving emotion regulation. Longitudinal data are needed to confirm aberrant developmental trajectories intra-individually and to examine whether these abnormalities are predictive of conversion to psychosis, and of later deficits in socioemotional functioning.

\section{Keywords}

psychosis; brain development; emotion; amygdala; prefrontal cortex; fMRI

\section{Introduction}

Patients with schizophrenia show impaired performance on tasks engaging a variety of emotion-related processes (e.g., perception, expression, regulation) (Fakra et al., 2008; Kohler et al., 2010; Kring \& Moran, 2008). Deficits in emotion processing are generally refractory to interventions (Harvey et al., 2006; Penn et al., 2009; Sergi et al., 2007) and are strongly related to social and occupational impairments, both contemporaneously and prospectively (Hooker \& Park, 2002; Kee et al., 2003; Mueser et al., 1996). Thus, understanding the timing of onset and neural underpinnings of emotion-related deficits could lead to more effective approaches to treatment and prevention of functional disabilities in schizophrenia.

Schizophrenia patients show decreased functional connectivity of the amygdala and prefrontal cortex during emotion processing (Fakra et al., 2008). Whether reduced functional connectivity is a cause or consequence of schizophrenia (or its treatment) is not yet clear. However, because individuals at clinical high risk (CHR) for psychosis exhibit emotionrelated behavioral deficits (Phillips \& Seidman, 2008; Addington et al., 2008; Kim et al., 2010; van Rijn et al., 2011) and healthy individuals with high psychosis-proneness display decreased cognitive control of emotion (Modinos, Ormel, \& Aleman, 2010), abnormal functional connectivity in networks subserving emotion processing might predate (and potentially contribute to) psychosis onset.

Disrupted neurodevelopmental processes resulting in reduced structural and functional brain connectivity are hypothesized to play a key role in the onset of schizophrenia (Karlsgodt et al., 2008; Lim et al., 1999; McGlashan \& Hoffman, 2000; Meyer-Lindenberg et al., 2001; Weinberger et al., 1994). Consistent with this view, CHR individuals who convert to psychosis display an increased rate of prefrontal gray matter contraction relative to nonconverting high-risk individuals (Pantelis et al., 2003; Sun et al., 2009), and CHR patients overall show an absence of age-related increases in white matter integrity compared with controls (Karlsgodt et al., 2009). While little work has focused on emotion processing, prior studies have demonstrated neural abnormalities associated with working memory and verbal fluency among CHR patients (Broome et al., 2009; Fusar-Poli et al., 2010; Smieskova et al., 2011).

The physiological consequences of structural brain maturation during adolescence involve fine-tuning of functional circuitry. Typically, a shift from short-range to long-range functional connectivity leads to separable networks and increased efficiency, with socioemotional networks achieving functional maturation later than sensory-motor networks (Fair et al., 2007; Kelly et al., 2009). Protracted development of networks subserving emotion regulation may derive from differential maturational timecourses of subcortical and 
prefrontal regions, increasing risk for emotion dysregulation during adolescence (Galvan et al., 2006). This circuitry is likely to be especially vulnerable among individuals with deficits in prefrontal structural integrity - i.e., CHR individuals who progress to full psychosis (e.g., Pantelis et al., 2003).

The present cross-sectional study investigated whether CHR patients display altered agerelated patterns of amygdala and prefrontal function. Brain activation was evaluated with functional magnetic resonance imaging (fMRI) during an emotional processing task (Lieberman et al., 2007). Prior work using this task has demonstrated robust amygdala and prefrontal activation, as well as evidence for the role of ventrolateral prefrontal cortex (vlPFC) in modulating amygdala reactivity during emotion labeling, a condition representing an incidental form of emotion regulation (Hariri et al., 2000; Lieberman et al., 2007).

Since the prefrontal-subcortical regulatory relationship becomes stronger (Casey et al., 2008) and activation in task-relevant regions increases across typical development (Durston et al., 2006), we expected increased vlPFC and decreased age-related amygdala activation among controls. We hypothesized that CHR patients would demonstrate decreased amygdala-prefrontal functional connectivity, as well as altered trajectories of amygdala and prefrontal activation, relative to controls. This pattern of findings would be consistent with a failure of prefrontal cortex to regulate amygdala reactivity, resulting in increased amygdala activity with increasing age.

\section{Materials and Methods}

\subsection{Participants}

Participants were 20 CHR adolescents and 14 age- and gender-matched healthy controls between 15 and 23 years old. The protocol was approved by Institutional Review Boards at the sites participating in the North American Prodrome Longitudinal Study (NAPLS), from which participants were drawn (Emory University, Harvard University, University of Calgary, University of California Los Angeles (UCLA), University of California San Diego, University of North Carolina (UNC), Yale University, Zucker Hillside Hospital), and participants provided informed consent or assent (parental informed consent for minors). The data in this report are from the initial qualifying phase of the fMRI component of the NAPLS consortium, in which four sites contributed scan data. This phase was used to collect preliminary data to evaluate the applicability of the present emotional processing paradigm in a sample of CHR youth. Based on results of this preliminary investigation, the paradigm has been implemented in the full NAPLS study, for which data collection is ongoing through 2013.

\subsection{Clinical Measures}

Participants were screened using the Structured Interview for Prodromal Syndromes (SIPS; McGlashan et al., 2001) for the presence of a prodromal syndrome: attenuated subthreshold psychotic symptoms, brief intermittent psychotic symptoms, substantial functional decline combined with genetic risk for psychosis, or schizotypal personality disorder in individuals $<18$ years old. CHR participants were excluded if they met DSM-IV criteria for an Axis I schizophrenia-spectrum disorder. Control participants were excluded if they met DSM-IV criteria for a psychiatric disorder, had a first-degree relative with a current or past psychotic disorder, or met prodromal criteria. General exclusions included substance dependence (past 6 months), neurological disorder, or Full Scale IQ $<70$. Overall functioning was measured using the Global Assessment of Functioning (GAF) scale. 


\section{3 fMRI Task Paradigm}

The experimental paradigm consisted of an emotional faces task (Hariri et al., 2000; Lieberman et al., 2007) (Figure 1). Participants viewed target faces or shapes while performing one of five tasks in each block of 10 trials (each trial was 5 seconds; i.e., 50second blocks in randomized order). Each condition type occurred once in each of two functional runs. Emotion labeling involved choosing which of two labels (e.g., "angry," "happy," "scared," "surprised") described a target face. Gender labeling involved selecting the gender-appropriate name for a target face. Emotion matching involved choosing which of two faces displayed the same emotion as a target face. Gender matching involved selecting which of two faces was the same gender as a target face. Shape matching involved selecting which of two shapes was the same as a target shape. Facial stimuli were chosen from a standardized set (Tottenham et al., 2009). Additional details appear elsewhere (Lieberman et al., 2007). Emotion labeling is considered to represent a form of incidental emotion regulation (Lieberman et al., 2007) and has been associated with decreases in negative emotion (Lieberman, Inagaki, Tabibnia, \& Crockett, 2011) and reduced physiological responsivity (Tabibnia, Lieberman, \& Craske, 2008). For this reason, we focused on emotion labeling in the present study, with appropriate contrasts (e.g., emotion labeling versus gender labeling, emotion labeling versus emotion matching) selected based on prior work (Lieberman et al., 2007).

\subsection{Functional Imaging Parameters}

Scanning was performed on Siemens Trio 3.0 Tesla (3T) scanners (UCLA, $\mathrm{n}=17$; Yale University, $\mathrm{n}=2$ ), a Siemens Allegra 3T scanner (UNC, n=14), and a GE 3T scanner (Zucker Hillside Hospital, $\mathrm{n}=1$ ). A standard radiofrequency head coil was employed. Anatomical reference scans were acquired to configure slice alignment. A T2-weighted image (0.9-mm in-plane resolution) was acquired using a set of high-resolution echo planar (EPI) localizers (Siemens: TR/TE 6310/67ms, 30 4-mm slices with 1-mm gap, 220-mm FOV; GE: TR/TE 6000/120ms, 30 4-mm slices with 1-mm gap, 220-mm FOV). Functional scans matched the AC-PC aligned T2 image and utilized an EPI sequence (TR/TE 2500/30ms, 77 degree flip angle, 30 4-mm slices). Each functional run consisted of 129 volumes.

\subsection{Image Processing}

Functional image analysis was performed using FSL (FMRIB's Software Library v. 4.0; Smith et al., 2004). Motion in EPI data was corrected using a six-parameter, rigid-body 3D co-registration (FLIRT), which registered each BOLD image to the middle data point in the timeseries. Data were registered for each participant (EPI to participant's T2-weighted structural image, then T2 to standard space brain) (Jenkinson \& Smith, 2001; Jenkinson et al., 2002). Data were spatially smoothed with a 5-mm (FWHM) Gaussian kernel and filtered with a non-linear high-pass filter (120s cut-off). Individual participant analyses employed FEAT (FMRI Expert Analysis Tool).

Timeseries statistical analysis on each participant was carried out using FILM (FMRIB's Improved Linear Model) with local autocorrelation correction. A univariate general linear model (GLM) was applied on a voxel-by-voxel basis such that each voxel's timeseries was individually fitted to the resulting model, with local autocorrelation correction applied within tissue type to improve temporal smoothness estimation (Smith et al., 2004; Woolrich et al., 2001). Each voxel's goodness-of-fit to the model was estimated; resulting parameter estimates indicated the degree to which signal change could be explained by each model. Each condition was modeled separately, with each correct trial modeled in its entirety in a block design fashion. Motion parameters were entered as covariates; participants with motion $>3 \mathrm{~mm}$ were excluded. 
Resulting contrast images were entered into second-level analyses (fixed effects model) to combine functional runs for each participant and to allow for inferences at the group level. Group analysis was carried out using FLAME (FMRIB's Local Analysis of Mixed Effects) (Behrens et al., 2003; Smith et al., 2004) with each participant's data, including parameter and variance estimates for each contrast from the lower-level analysis. To correct for multiple comparisons, resulting Z-statistic images were thresholded using clusters determined by $\mathrm{Z}>2.3$ and a corrected cluster significance threshold of $\mathrm{p}=0.05$ (Forman et al., 1995; Friston et al., 1994; Worsley et al., 1992). Cluster p-values were determined using spatial smoothness estimation in FEAT.

\subsection{Regions of Interest}

Regions of interest (ROI) were selected based on prior work (Lieberman et al., 2007). An anatomically-defined left amygdala mask was defined using the Harvard-Oxford Structural Atlas (Kennedy et al., 1998; Makris et al., 1999). Voxels with atlas-derived values corresponding to a $\geq 25 \%$ probability of belonging to the left amygdala were included. For the vlPFC ROI, a spherical (diameter $=10 \mathrm{~mm}$ ) mask was created with center at coordinates for peak vlPFC activation $(56,22,8)$ during emotion labeling versus gender labeling among controls in prior work (Lieberman et al., 2007) (Figure 2). FSL's Featquery was used to warp ROIs back into each participant's space by applying the inverse of the transformation matrix used during the initial registration. The motion-corrected, smoothed, and filtered data were probed for mean percent signal change during emotion labeling versus emotion matching in the left amygdala and during emotion labeling versus gender labeling in the right vlPFC. These contrasts for the amygdala and vlPFC were selected based on their use in research with the same task in healthy controls (Hariri et al., 2000; Lieberman et al., 2007).

\subsection{Age-Related Analyses of Functional Activation}

A GLM was constructed to model age for each group while covarying for sex. This analysis allowed for examination of an interaction by producing statistical maps of regions for which activation increased for patients while decreasing with age for controls, or vice versa (see Supplemental Methods and Results). Following group-level analyses in FSL, an ROI approach was used to examine age-related amygdala and vlPFC activation in each group. For each ROI, a regression analysis was conducted in SPSS 18.0 predicting percent signal change based on age, group, a group $\times$ age interaction, and sex. The interaction term was used to examine whether age trajectories of functional activation differed between groups. To avoid effects of outliers, participants with a studentized residual or studentized deleted residual $<-3$ or $>3$ were removed, resulting in exclusion of one CHR participant.

We also tested whether site significantly predicted activation results in ROI analyses of the amygdala and vlPFC or functional connectivity results in the psychophysiological interaction (PPI) analysis conducted across all voxels in the brain. No differences were found between sites, thus site was removed from models to preserve degrees of freedom.

Initial between-group analyses were conducted to compare whole-brain activation of patients and controls, regardless of age. In general, patients showed a pattern of hypoactivation across relevant task contrasts (see Supplemental Methods and Results). However, the group effects were not particularly robust, and between-group ROI analyses of the amygdala and vlPFC were not significant, because analyses of developmental trajectories revealed that the direction of patient and control differences varied across the age continuum. 


\subsection{Psychophysiological Interaction Analysis}

A PPI analysis (Friston et al., 1997) was conducted to examine whether task-dependent functional connectivity between the amygdala and prefrontal regions differed between groups. That is, the PPI analysis tested whether CHR patients and controls differed on the extent to which the amygdala covaried with other brain regions more during emotion labeling than during emotion matching. Emotion labeling versus emotion matching was chosen as the task contrast of interest because prior studies in healthy individuals have demonstrated stronger amygdala-prefrontal coupling during emotion labeling than emotion matching (Lieberman et al., 2007).

The GLM analysis was carried out in FSL with regressors for task, seed region timeseries, and the interaction of task and timeseries. The psychological (task condition) regressor modeled whether a given trial consisted of emotion labeling or emotion matching. The physiological (seed region timeseries) regressor comprised the timeseries for the left amygdala. A third regressor modeled the interaction of the psychological regressor and the physiological regressor, such that it identified regions that covaried in a task-dependent manner with the amygdala (i.e., regions that significantly correlated more with the amygdala during emotion labeling than during emotion matching).

As in the prior analyses of functional activation, a first level analysis was conducted for each individual participant to identify regions that significantly covaried with the amygdala in a task-dependent manner for each run. A second level analysis combined results from the first and second run for each participant. Finally, a group level analysis identified differences between CHR patients and controls in terms of regions that significantly covaried with the amygdala more during emotion labeling than during emotion matching. Group analysis was carried out using FLAME (Behrens et al., 2003; Smith et al., 2004), and resulting Z-statistic images were thresholded using clusters determined by $Z>2.3$ and a corrected cluster significance threshold of $\mathrm{p}=0.05$ to correct for multiple comparisons (Forman et al., 1995; Friston et al., 1994; Worsley et al., 1992).

\subsection{Demographic and Behavioral Data Analysis}

Independent sample t-tests or chi-square tests were used to compare age, gender, GAF score, and task performance (accuracy, reaction time (RT)) between patients and controls, as appropriate. Paired sample t-tests compared mean accuracy and mean RT between conditions across all participants. Correlational analyses tested whether age was associated with performance.

\section{Results}

\subsection{Participant Characteristics}

The CHR and control groups did not differ on age $(\mathrm{p}=.913)$ or sex $\left(\mathrm{X}^{2}=.68, \mathrm{p}=.410\right)$. As shown in Table 1, the majority of CHR patients (17/20) met criteria for Attenuated Positive Symptoms. Patients exhibited significantly lower overall functioning (GAF) relative to controls ( $\mathrm{p}<.001)$. Two of the 20 CHR participants had converted to psychosis $(10 \%)$ at the time of this analysis; since most of the subjects had not yet been followed for more than 6 months, several more cases would be expected to convert over the next few years. Given the number of conversions to date, it was not possible to conduct analyses comparing activation or functional connectivity for converting versus non-converting CHR participants.

\subsection{Behavioral Task Performance}

Participants performed the task with a mean accuracy of $92.8 \%$ across conditions (Table 2). Patients and controls did not significantly differ on accuracy overall or on any condition. 
Overall, participants displayed greater accuracy for gender labeling versus emotion matching $(\mathrm{p}=.004)$ and emotion labeling $(\mathrm{p}=.025)$ and for shape matching versus emotion matching $(\mathrm{p}=.036)$. Patients and controls did not differ in RT overall or on any condition (Table 3). Overall, participants responded faster during shape matching than any other condition ( $\mathrm{p}<.050)$. Mean RT was faster for gender matching than gender labeling, emotion matching, and emotion labeling ( $\mathrm{p}<.001)$, for gender labeling versus emotion labeling or emotion matching $(\mathrm{p}<.001)$, and for emotion labeling versus emotion matching $(\mathrm{p}<.001)$. Age and site did not significantly relate to RT or accuracy.

\subsection{Age-Related vIPFC Functional Activation}

Regression analysis revealed a significant group $\times$ age interaction in the right vlPFC $(\mathrm{F}(4,29)=5.43, \mathrm{p}=.027)$, such that age-related trajectories of functional activation during emotion labeling versus gender labeling differed between CHR patients and controls (Figure 3). Controls showed increasing vlPFC activation with increasing age, whereas CHR patients showed decreasing vlPFC activation with increasing age. There were no significant main effects of group, age, or sex (Table 4).

\subsection{Age-Related Amygdala Functional Activation}

There was a significant group $\times$ age interaction for functional activation during emotion labeling versus emotion matching in the left amygdala $(F(4,28)=4.84, p=.037$; Figure 4). This analysis demonstrated an opposite pattern of age-related trajectories for the amygdala compared with vlPFC. Controls showed decreasing amygdala activation with increasing age, whereas CHR patients showed increasing amygdala activation with increasing age. There were no significant main effects of group, age, or sex (Table 5).

\subsection{Functional Connectivity between Amygdala and Prefrontal Regions}

Task-dependent functional connectivity differed between CHR patients and controls, such that patients exhibited weaker inverse amygdala-prefrontal covariation relative to controls for emotion labeling relative to emotion matching. In a whole-brain analysis, this effect was observed in the left orbitofrontal gyrus and left inferior frontal gyrus $(\mathrm{Z}=3.82, \mathrm{p}=.027$, corrected) (Figure 5). The peak of covariation with the amygdala was at coordinates -50 , $32,-10$. When statistical maps of covariation with the amygdala were examined for each group separately, no regions significantly covaried with the amygdala in the CHR group.

\subsection{Medication Use}

In order to examine whether medication use influenced findings, we examined the effects of antipsychotic and antidepressant use in separate analyses. Functional activation and connectivity did not significantly relate to use of either class of drug, and all findings remained significant over and above medication status (see Supplemental Methods and Results).

\section{Discussion}

The present study examined age-related trajectories of neural circuitry subserving emotion regulation in adolescents at CHR for psychosis and typically developing adolescents. The findings indicated that CHR adolescents display altered age-related differences in functional activation in amygdala and prefrontal regions relative to controls, suggesting that abnormalities in emotion-related neural circuitry may exist prior to illness onset and may emerge with development over time. In addition, CHR adolescents exhibited decreased amygdala-prefrontal functional connectivity, consistent with findings of disconnectivity in schizophrenia. To our knowledge, this is the first study to examine functional connectivity 
and trajectories of functional activation in CHR subjects in regions important to emotion processing. These findings provide novel insight into disrupted amygdala-prefrontal interactions and the possible developmental course of these abnormalities in at-risk patients.

Age-related differences in functional activation observed among controls were consistent with expected development. We selected a circumscribed prefrontal region shown to be important for emotion labeling (Lieberman et al., 2007); the pattern of increasing vlPFC activation with age is consistent with prior evidence of a generally increased magnitude of activation in task-relevant regions with age (Durston et al., 2006; Pfeifer et al., 2011). By contrast, there was a pattern of reduced amygdala activation with age; this effect may derive from enhanced regulatory control and increasing strength of functional circuitry, as networks subserving emotion regulation continue to develop throughout adolescence (Kelly et al., 2009). Though research has demonstrated a general developmental shift from diffuse to focal activation (e.g., Durston et al., 2006), there might also be differences in global versus task-specific activation changes, as well as different age-related activation patterns depending on task domain (e.g., emotional versus executive function).

In contrast to age-related patterns among controls, CHR adolescents exhibited decreased vlPFC activation and increased amygdala activation with age, consistent with emotion dysregulation, and weaker inverse amygdala-vlPFC functional connectivity, supporting the relevance of disconnectivity to psychosis onset. Disruptions in structural and functional connectivity may prevent adequate regulation of amygdala activity. A similar pattern was seen in a study of adults with schizophrenia; patients displayed greater amygdala reactivity, as well as decreased amygdala-prefrontal functional connectivity (Fakra et al., 2008).

Understanding the nature of physiological systems subserving emotion processing has important implications. Emotion-related deficits in schizophrenia interfere with socioemotional functioning, social cognition, and functional outcomes (Hooker \& Park, 2002; Kee et al., 2003; Pinkham et al., 2003). As such, continued emotional disturbances may contribute to ongoing functional impairment in schizophrenia even after positive symptoms are treated. The present findings suggest that CHR patients exhibit alteration in an incidental form of emotion regulation (i.e., emotion labeling). Though individuals are not asked to try to regulate their emotions during emotion labeling, prior research has demonstrated that this process is associated with common effects of emotion regulation, including reductions in self-reported distress, decreased physiological reactivity, increased vlPFC activation, decreased amygdala activity, and negatively correlated prefrontal and amygdala responses (Hariri, Bookheimer, \& Mazziotta, 2000; Lieberman et al., 2007; Tabibnia, Lieberman, \& Craske, 2008; Payer, Lieberman, \& London, 2011; Lieberman, Inagaki, Tabibnia, \& Crockett, 2011). While future research is necessary to elucidate the functional implications of this implicit psychological process, prior work on emotion labeling demonstrating its effects of reducing subjective reports of negative emotion (Lieberman, Inagaki, Tabibnia, \& Crockett, 2011) and reducing physiological reactivity (Tabibnia, Lieberman, \& Craske, 2008) suggests that alterations in this process and in amygdala-vlPFC circuitry may have important consequences for patients at risk for psychosis.

The psychosis prodrome allows for a unique examination of emotion-related neural circuitry prior to overt illness, while minimizing common confounds (e.g., long-term antipsychotic treatment). Given the heterogeneous outcomes of CHR participants (Cannon et al., 2008), future work would benefit from comparing converters and non-converters. This study is limited by several factors to be addressed in future research. First, the sample size is relatively modest, as the data were collected as part of an initial phase of a larger study. Results indicate that the emotional processing paradigm is appropriate for use in the CHR 
population and support the decision to incorporate this paradigm in ongoing NAPLS data collection. Follow-up research with larger samples will be critical for elucidating developmental changes in amygdala-prefrontal interactions. It is important to note, however, that in general small sample size is problematic due to low power to detect statistical effects; in this case, power was clearly sufficient to detect numerous effects of interest. Second, our data are cross-sectional; thus, the present results do not represent within-subject developmental trajectories, and it will be important to examine whether these results replicate in longitudinal follow-up data. Third, a subset of CHR participants has been exposed to medication; however, there was no evidence that medication use influenced the current findings, as the measures of functional activation and connectivity did not relate to medication use. Fourth, though we did not detect between-site differences, it will be important to address the issue of multi-site data collection in larger samples with increased power to test for site-specific effects. We re-analyzed the present data excluding participants from Yale University and Zucker Hillside Hospital and observed the same results (see Supplemental Methods and Results). Finally, the CHR group represents a clinically heterogeneous sample, and only a subset of the CHR sample is likely to develop psychosis. Thus, future work comparing converters with non-converters will greatly enhance knowledge of the relationship between amygdala-prefrontal circuitry and later psychosis.

We did not find group- or age-related performance differences, suggesting that observed differences in brain activation were not secondary to behavioral differences. Although prior research has demonstrated altered performance among schizophrenia patients (Fakra et al., 2008), CHR individuals may not yet exhibit performance differences on this task. Changes in brain activation may precede or predict behavioral deterioration. The lack of performance differences may also relate to the sample's heterogeneous nature (e.g., not all CHR participants will develop psychosis).

Our findings are consistent with the hypothesis that patients at CHR for psychosis display altered trajectories of neurodevelopment in the amygdala and prefrontal cortex, as well as decreased functional connectivity among regions subserving emotion regulation, relative to controls. Given the debilitating socioemotional deficits associated with schizophrenia, the present findings provide critical insight into emotion-related abnormalities in neural circuitry that are present prior to overt psychosis. Moreover, knowledge about early disruptions in amygdala-prefrontal interactions may enhance prediction of later socioemotional functioning and conversion to psychosis.

\section{Supplementary Material}

Refer to Web version on PubMed Central for supplementary material.

\section{Acknowledgments}

The authors would like to thank the International Mental Health Research Organization and the Staglin family for their generosity and support for this research.

\section{References}

Addington J, Penn D, Woods SW, Addington D, Perkins DO. Facial affect recognition in individuals at clinical high risk for psychosis. The British Journal of Psychiatry. 2008; 192(1):67-68. [PubMed: 18174514]

Behrens TE, Johansen-Berg H, Woolrich MW, Smith SM, Wheeler-Kingshott CA, Boulby PA, et al. Non-invasive mapping of connections between human thalamus and cortex using diffusion imaging. Nature Neuroscience. 2003; 6(7):750-757. 
Broome MR, Matthiasson P, Fusar-Poli P, Woolley JB, Johns LC, Tabraham P, et al. Neural correlates of executive function and working memory in the 'at-risk mental state'. The British Journal of Psychiatry. 2009; 194:25-33. [PubMed: 19118321]

Cannon TD, Cadenhead K, Cornblatt B, Woods SW, Addington J, Walker E, et al. Prediction of psychosis in youth at clinical high risk: a multisite longitudinal study in North America. Archives of General Psychiatry. 2008; 65:28-37. [PubMed: 18180426]

Casey BJ, Getz S, Galvan A. The adolescent brain. Developmental Review. 2008; 28(1):62-77. [PubMed: 18688292]

Durston S, Davidson MC, Tottenham N, Galvan A, Spicer J, Fossella JA, et al. A shift from diffuse to focal cortical activity with development. Developmental Science. 2006; 9(1):1-8. [PubMed: 16445387]

Fair DA, Dosenbach NU, Church JA, Cohen AL, Brahmbhatt S, Miezin FM, et al. Development of distinct control networks through segregation and integration. Proceedings of the National Academy of Sciences. 2007; 104(33):13507-13512.

Fakra E, Salgado-Pineda P, Delaveau P, Hariri AR, Blin O. Neural bases of different cognitive strategies for facial affect processing in schizophrenia. Schizophrenia Research. 2008; 100(1-3): 191-205. [PubMed: 18234477]

Forman SD, Cohen JD, Fitzgerald M, Eddy WF, Mintum MA, Noll DC. Improved assessment of significant activation in functional magnetic resonance imaging (fMRI): use of a cluster-size threshold. Magnetic Resonance Medicine. 1995; 33(5):636-647.

Friston KJ, Worsley K, Frackowiak R, Mazziotta JC, Evans AC. Assessing the significance of focal activations using their spatial extent. Human Brain Mapping. 1994; 1:214-220.

Friston KJ, Buechel C, Fink GR, Morris J, Rolls E, Dolan RJ. Psychophysiological and modulatory interactions in neuroimaging. Neuroimage. 1997; 6:218-229. [PubMed: 9344826]

Fusar-Poli P, Broome MR, Matthiasson P, Woolley JB, Johns LC, Tabraham P, et al. Spatial working memory in individuals at high risk for psychosis: Longitudinal fMRI study. Schizophrenia Research. 2010; 123:45-52. [PubMed: 20688479]

Galvan A, Hare TA, Parra CE, Penn J, Voss H, Glover G, et al. Earlier development of the accumbens relative to orbitofrontal cortex might underlie risk-taking behavior in adolescents. Journal of Neuroscience. 2006; 26(25):6885-6892. [PubMed: 16793895]

Hariri AR, Bookheimer SY, Mazziotta JC. Modulating emotional responses: effects of a neocortical network on the limbic system. Neuroreport. 2000; 11:43-48. [PubMed: 10683827]

Harvey PD, Patterson TL, Potter LS, Zhong K, Brecher M. Improvement in social competence with short-term atypical antipsychotic treatment: a randomized, double-blind comparison of quetiapine versus risperidone for social competence, social cognition, and neuropsychological functioning. American Journal of Psychiatry. 2006; 163(11):1918-1925. [PubMed: 17074943]

Hooker C, Park S. Emotion processing and its relationship to social functioning in schizophrenia patients. Psychiatry Research. 2002; 112:41-50. [PubMed: 12379449]

Jenkinson M, Smith S. A global optimisation method for robust affine registration of brain images. Medical Image Analysis. 2001; 5:143-156. [PubMed: 11516708]

Jenkinson M, Bannister P, Brady M, Smith S. Improved optimization for the robust and accurate linear registration and motion correction of brain images. NeuroImage. 2002; 17:825-841. [PubMed: 12377157]

Karlsgodt KH, Sun D, Jimenez AM, Lutkenhoff ES, Willhite R, van Erp TGM, et al. Developmental disruptions in neural connectivity in the pathophysiology of schizophrenia. Development and Psychopathology. 2008; 20:1297-1327. [PubMed: 18838043]

Karlsgodt KH, Niendam TA, Bearden CE, Cannon TD. White matter integrity and prediction of social and role functioning in subjects at ultra-high risk for psychosis. Biological Psychiatry. 2009; 66(6):562-569. [PubMed: 19423081]

Kee KS, Green MF, Mintz J, Brekke JS. Is emotion processing a predictor of functional outcome in schizophrenia? Schizophrenia Bulletin. 2003; 29(3):487-497. [PubMed: 14609242]

Kelly AM, Di Martino A, Uddin LQ, Shehzad Z, Gee DG, Reiss PT, et al. Development of anterior cingulate functional connectivity from late childhood to early adulthood. Cerebral Cortex. 2009; 19(3):640-657. [PubMed: 18653667] 
Kennedy DN, Lange N, Makris N, Bates J, Meyer J, Caviness VS. Gyri of the human neocortex: An MRI-based analysis of volume and variance. Cerebral Cortex. 1998; 8:372-384. [PubMed: 9651132]

Kim HS, Shin NY, Choi JS, Jung MH, Jang JH, Kang DH, et al. Processing of facial configuration in individuals at ultra-high risk for schizophrenia. Schizophrenia Research. 2010; 118(1-3):81-87. [PubMed: 20133107]

Kohler CG, Walker JB, Martin EA, Healey KM, Moberg PJ. Facial emotion perception in schizophrenia: A meta-analytic review. Schizophrenia Bulletin. 2010; 36(5):1009-1019. [PubMed: 19329561]

Kring AM, Moran EK. Emotional response deficits in schizophrenia: Insights from affective science. Schizophrenia Bulletin. 2008; 34(5):819-834. [PubMed: 18579556]

Lieberman MD, Eisenberger NI, Crockett MJ, Tom SM, Pfeifer JH, Way BM. Putting feelings into words: Affect labeling disrupts amygdala activity in response to affective stimuli. Psychological Science. 2007; 18(5):421-428. [PubMed: 17576282]

Lieberman MD, Inagaki TK, Tabibnia G, Crockett MJ. Subjective responses to emotional stimuli during labeling, reappraisal, and distraction. Emotion. 2011; 11(3):468-480. [PubMed: 21534661]

Lim KO, Hedehus M, Moseley M, de Crespigny A, Sullivan EV, Pfefferbaum A. Compromised white matter tract integrity in schizophrenia inferred from diffusion tensor imaging. Archives of General Psychiatry. 1999; 56(4):367-374. [PubMed: 10197834]

Makris N, Meyer JW, Bates JF, Yeterian EH, Kennedy DN, Caviness VS. MRI-Based topographic parcellation of human cerebral white matter and nuclei II. Rationale and applications with systematics of cerebral connectivity. NeuroImage. 1999; 9:18-45. [PubMed: 9918726]

McGlashan TH, Hoffman RE. Schizophrenia as a disorder of developmentally reduced synaptic connectivity. Archives of General Psychiatry. 2000; 57:637-648. [PubMed: 10891034]

McGlashan, TH.; Miller, TJ.; Woods, SW.; Rosen, JL.; Hoffman, RE.; Davidson, L. Structured Clinical Interview for Prodromal Syndromes. Yale School of Medicine: PRIME Research Clinic; 2001.

Meyer-Lindenberg A, Poline J, Kohn PD, Holt JL, Egan MF, Weinberger DR, et al. Evidence for abnormal cortical functional connectivity during working memory in schizophrenia. American Journal of Psychiatry. 2001; 158:1809-1817. [PubMed: 11691686]

Modinos G, Ormel J, Aleman A. Altered activation and functional connectivity of neural systems supporting cognitive control of emotion in psychosis proneness. Schizophrenia Research. 2010; 118(1-3):88-97. [PubMed: 20188516]

Mueser KT, Doonan R, Penn DL, Blanchard JJ, Bellack AS, Nishith P, et al. Emotion recognition and social competence in chronic schizophrenia. Journal of Abnormal Psychology. 1996; 105(2):271275. [PubMed: 8723008]

Pantelis C, Velakoulis D, McGorry P, Wood SJ, Suckling J, Phillips LJ, et al. Neuroanatomical abnormalities before and after onset of psychosis: a cross-sectional and longitudinal MRI comparison. Lancet. 2003; 361:281-288. [PubMed: 12559861]

Payer DE, Lieberman MD, London ED. Neural correlates of affect processing and aggression in methamphetamine dependence. Archives of General Psychiatry. 2011; 68(3):271-282. [PubMed: 21041607]

Pfeifer JH, Masten CL, Moore WE, Oswald TM, Mazziotta JC, et al. Entering adolescence: resistance to peer influence, risky behavior, and neural changes in emotion reactivity. Neuron. 2011; 69(5): 1029-1036. [PubMed: 21382560]

Penn DL, Keefe RSE, Davis SM, Meyer PS, Perkins DO, Losardo D, et al. The effects of antipsychotic medications on emotion perception in patients with chronic schizophrenia in the CATIE trial. Schizophrenia Research. 2009; 115(1):17-23. [PubMed: 19766459]

Phillips LK, Seidman LJ. Emotion processing in persons at risk for schizophrenia. Schizophrenia Bulletin. 2008; 34(5):888-903. [PubMed: 18644853]

Pinkham A, Penn DL, Perkins DO, Lieberman J. Implications for the neural basis of social cognition for the study of schizophrenia. American Journal of Psychiatry. 2003; 160:815-824. [PubMed: 12727681] 
Sergi MJ, Green MF, Widmark C, Reist C, Erhart S, Braff DL, et al. Social cognition and neurocognition: effects of risperidone, olanzapine, and haloperidol. American Journal of Psychiatry. 2007; 164(10):1585-1592. [PubMed: 17898351]

Smieskova R, Allen P, Simon A, Aston J, Bendfeldt K, Drewe J, et al. Different duration of at-risk mental state associated with neurofunctional abnormalities. A multimodal imaging study. Human Brain Mapping. in press.

Smith SM, Jenkinson M, Woolrich MW, Beckmann CF, Behrens TE, Johansen-Berg H, et al. Advances in functional and structural MR image analysis and implementation in FSL. NeuroImage. 2004; 23:s208-s219. [PubMed: 15501092]

Sun D, Phillips L, Velakoulis D, Yung A, McGorry PD, Wood SJ, et al. Progressive brain structural changes mapped as psychosis develops in 'at risk' individuals. Schizophrenia Research. 2009; 108(1-3):85-92. [PubMed: 19138834]

Tabibnia G, Lieberman MD, Craske MG. The lasting effect of words on feelings: Words may facilitate exposure effects to threatening images. Emotion. 2008; 8(3):307-317. [PubMed: 18540747]

Tottenham N, Tanaka JW, Leon AC, McCarry T, Nurse M, Hare TA, et al. The NimStim set of facial expressions: judgments from untrained research participants. Psychiatry Research. 2009; 168(3): 242-249. [PubMed: 19564050]

van Rijn S, Aleman A, de Sonneville L, Sprong M, Ziermans T, Schothorst P, et al. Misattribution of facial expressions of emotion in adolescents at increased risk of psychosis: the role of inhibitory control. Psychological Medicine. 2011; 41(3):499-508. [PubMed: 20507669]

Weinberger DR, Aloia MS, Goldberg TE, Berman KF. The frontal lobes and schizophrenia. Journal of Neuropsychiatry and Clinical Neurosciences. 1994; 6(4):419-427. [PubMed: 7841813]

Woolrich MW, Ripley BD, Brady M, Smith SM. Temporal autocorrelation in univariate linear modeling of fMRI data. NeuroImage. 2001; 14(6):1370-1386. [PubMed: 11707093]

Worsley KJ, Evans AC, Marrett S, Neelin P. A three-dimensional statistical analysis for CBF activation studies in human brain. Journal of Cerebral Blood Flow Metabolism. 1992; 12(6):900918. [PubMed: 1400644]

\section{Appendix. The North American Prodrome Longitudinal Study (NAPLS) Consortium}

\section{Executive Committee}

Tyrone D. Cannon (UCLA, Chair), Jean Addington (Calgary), Kristin Cadenhead (UCSD), Barbara Cornblatt (Zucker Hillside), Robert Heinssen (NIMH), Daniel Mathalon (UCSF), Thomas McGlashan (Yale), Diana Perkins (UNC), Judy Rumsey (NIMH), Larry Seidman (Harvard), Ming Tsuang (UCSD), Elaine Walker (Emory), Scott W. Woods (Yale)

\section{Study Personnel by Site University of California, Los Angeles}

Principal Investigator: Tyrone D. Cannon

Clinical/Neurocognitive Investigator: Carrie E. Bearden

Neuroimaging Investigators: Sarah Jacobson, Frank Sun, Katherine Karlsgodt, Dylan Gee, Jennifer Forsyth, Arthur Toga, Paul Thompson

Electrophysiology Investigator: Peter Bachman

Study Coordinator/Database Manager: Jamie Zinberg

Recruitment: Sandra De Silva 
Scheduling: Angela Andaya

Administrative Assistant: Cristina Roman

Assessors: Shauna McManus, Sarah Marvin, Miguel Villodas

Research Assistants: Nichol Ferng, Anna Xu, Wendy Lau

\section{Emory University}

Principal Investigator: Elaine Walker

Clinical/Neurocognitive Investigator: Hanan Trotman

Neuroimaging Investigators: Xiaoping Hu, Lei Zhou, Stephan Hamann, Arthur Ryan

Electrophysiology Investigator: Erica Duncan

Study Coordinator/Database Manager: Joy Brasfield

Recruitment: Arthur Ryan

Scheduling: Anna Pless

Administrative Assistant: Ben Perlow

Assessors: Dan Shapiro, Sandy Goulding, Carrie Holtzman, Allison MacDonald, Arthur Ryan, Erin Jones

Research Assistants: David Lui, Ben Perlow

\section{Harvard University/Beth Israel Deaconess Medical Center}

Principal Investigator: Larry Seidman

Clinical Investigator: Kristen Woodberry, Anthony Giuliano, Michelle Friedman-Yakoobian

Neurocognitive Investigator: William Stone

Neuroimaging Investigator: Heidi Thermenos

Electrophysiology Investigator: Margaret Niznikiewicz, Robert McCarley

Database Manager: Linda Tucker

Recruitment: Corin Pilo

Administrative Assistant: Maryan Picard

Assessors: Benjamin Brent, Ann Cousins, Raquelle Mesholam-Gately, Andrea Gnong Granato, Janine Rodenhiser-Hill, Joanne Wojcik

Research Assistants: Lauren Gibson, Richard Juelich, Danbee Kim, Grace Min, Rachel Serur, Beril Yaffe

\section{Zucker Hillside Hospital}

Principal Investigator: Barbara Cornblatt 
Clinical Investigator: Andrea Auther

Neurocognitive Investigator: Danielle McLaughlin

Neuroimaging Investigators: Doreen Olvet, Peter Kingsley

Electrophysiology Investigator: Ricardo Carrion

Recruitment: Gary Brucato

Scheduling: Ruth Olsen

Administrative Assistant: Tricia Taylor

Assessors: Rita Barsky, Stephanie Snyder, Miranda Farabaugh

Research Assistant: Jeremy Chang

FFT Therapist: Kristin Candan

\section{University of North Carolina-Chapel Hill}

Principal Investigator: Diana Perkins

Clinical/Neurocognitive Investigator: David Penn

Neuroimaging Investigators: Ayse Belger

Electrophysiology Investigator: Franc Donkers

Study Coordinator/Database Manager: Andrea Pelletier

Recruitment: Karen Graham, Bryan Landaas

Scheduling: Andrea Pelletier

Administrative Assistant: Ellen Rothman

Assessors: Jennifer Nieri, Bryan Landaas

Research Assistants: Katie Lansing, Andrea Pelletier

\section{University of California, San Diego}

Principal Investigator: Kristin Cadenhead

Neurocognitive Investigator: Robert Heaton

Neuroimaging Investigators: Greg Brown, Heline Mirzakhanian

Electrophysiology Investigator: Greg Light

Study Coordinator/Recruitment/Clinical Assessment: Tracy Alderman

Recruitment/Clinical Assessment: Isabel Domingues, Nasra Haroun

Scheduling/Database: Steve Reding 
Research Assistants: Jason Nunag, Daniel Roman, Clara Robles

\section{University of Calgary}

Principal Investigator: Jean Addington

Genetics Investigator: Jim Kennedy

Neuroimaging Investigators: Richard Frayne, Brad Goodyear, Thomas Raedler

Electrophysiology Investigator: Neelan Pillay

Study Coordinator: Jacque Stowkowy

Database Developer: Jack Addington

Database Manager: Lu Lui

Neurocognitive and ERP coordinator: Danijela Piskulic

Recruitment: Lisa McGregor, Angie Kumar

Assessors: Catherine Marshall, Nora MacQuarrie, Kendra Smith, Emma Fitton, Erin Falukozi

Research Assistants: Mark Colijn, Aaron Peterson, Sona Sandu, Lianne Legere

\section{Yale University}

Principal Investigator: Scott W. Woods

Co-Principal Investigator: Thomas H. McGlashan

Neurocognitive Investigator: Keith A. Hawkins

Neuroimaging Investigators: Maolin Qiu, R. Todd Constable, Godfrey D. Pearlson

Electrophysiology Investigator: Jason K. Johannesen, Handan Gunduz-Bruce

Study Coordinator/Database Manager: John R. Saksa

Recruitment: Barbara C. Walsh

Assessor: Nicole Popp-Santamauro

Research Assistants: Joshua Kenney, Brenton Roman, Andrew Carlquist

\section{University of California, San Francisco}

Principal Investigator: Daniel Mathalon

Study Manager: Brian J. Roach

Data Analyst: Ryan M. Miller

\section{National Institute of Mental Health}

Scientific Project Officer: Robert Heinssen 
Program Officer: Judy Rumsey 


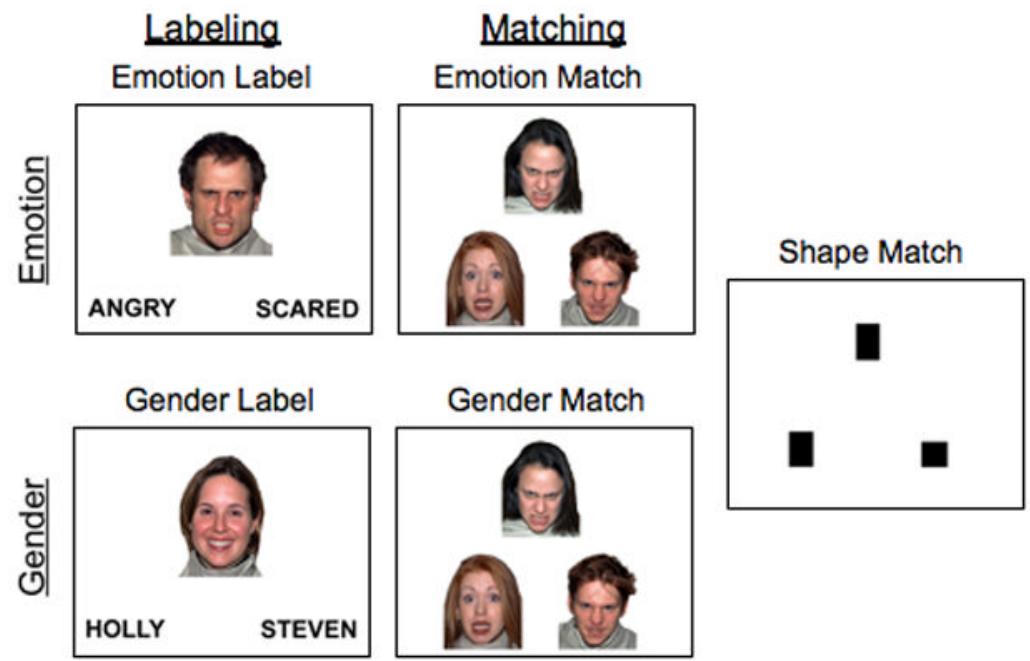

Figure 1. Task Design

The emotional faces fMRI task consisted of Emotion Labeling and Emotion Matching, as well as non-emotional control conditions of Gender Labeling, Gender Matching, and Shape Matching (adapted from Lieberman et al., 2007). 


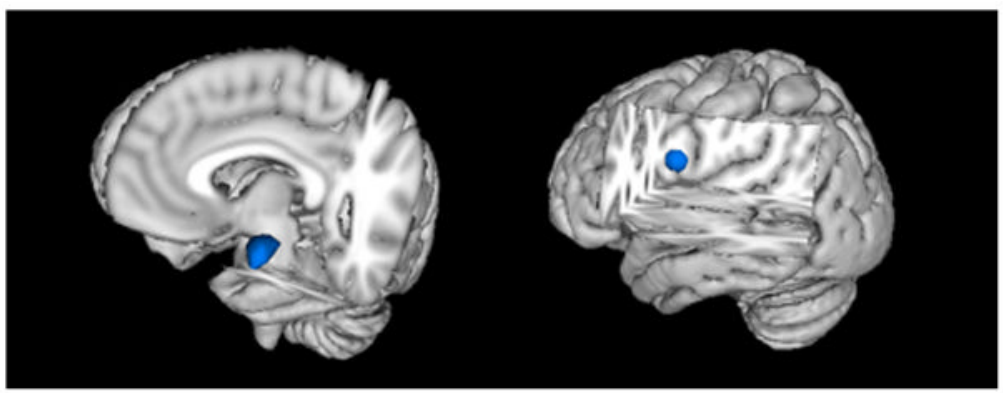

Figure 2. Regions of Interest

Regions of interest for the left amygdala (left) and right vlPFC (right) are shown. For the left amygdala, an anatomical ROI was used based on the probabilistic Harvard-Oxford Structural Atlas. The right vlPFC ROI mask was created using a spherical ROI centered at $56,22,8$, a peak of activation for emotion labeling versus gender labeling in a study of healthy controls (Lieberman et al., 2007). 


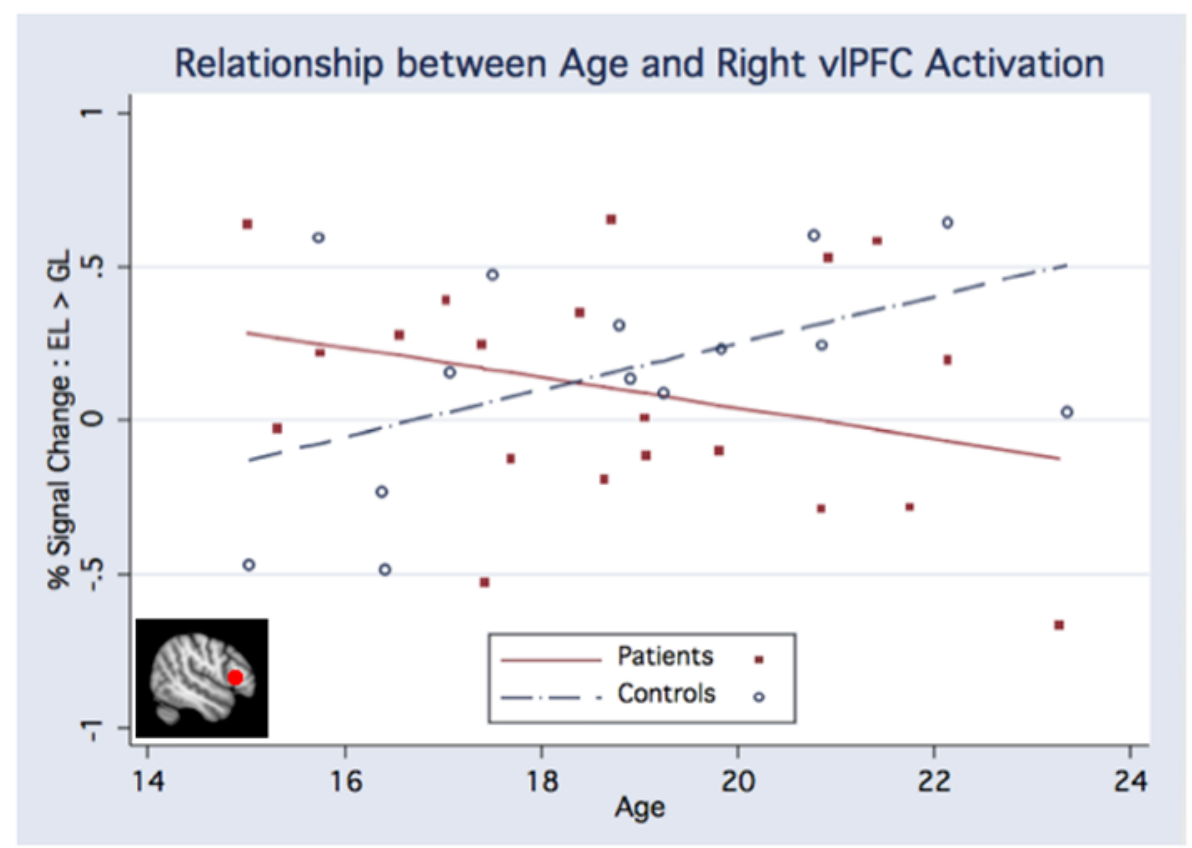

Figure 3. vlPFC Trajectories

Analysis of the right vlPFC revealed a significant interaction $(F(4,29)=5.43, p=.027)$ between age-related trajectories of functional activation during emotion labeling versus gender labeling for CHR patients and controls, such that controls showed increasing vlPFC activation whereas CHR patients showed decreasing vlPFC activation across development. 


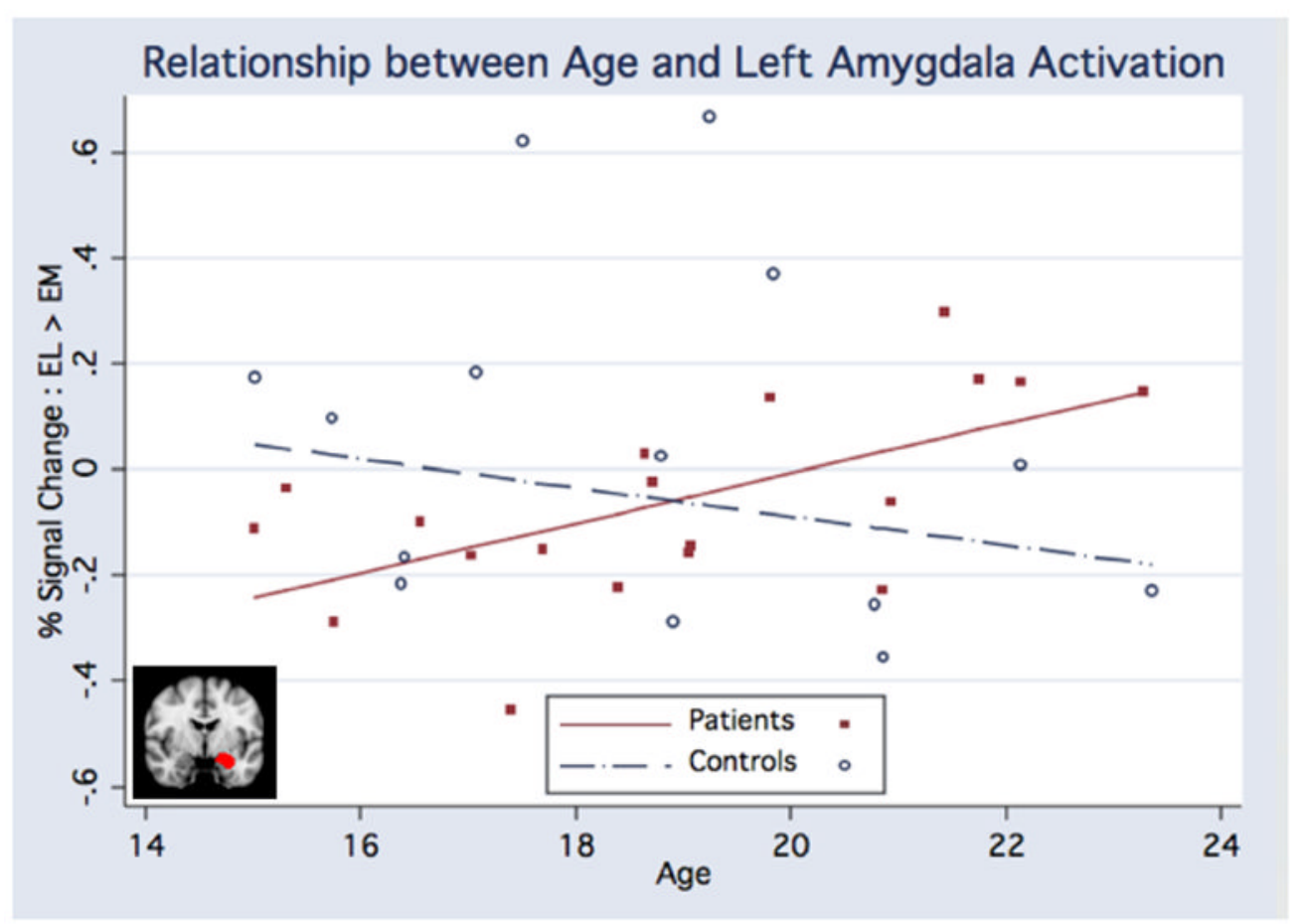

Figure 4. Amygdala Trajectories

Regression analysis demonstrated a significant interaction $(F(4,28)=4.84, p=.037)$ between age-related trajectories of left amygdala activation during emotion labeling versus emotion matching for CHR patients and controls, such that controls exhibited decreasing amygdala activation whereas CHR patients showed increasing amygdala activation with increasing age. 


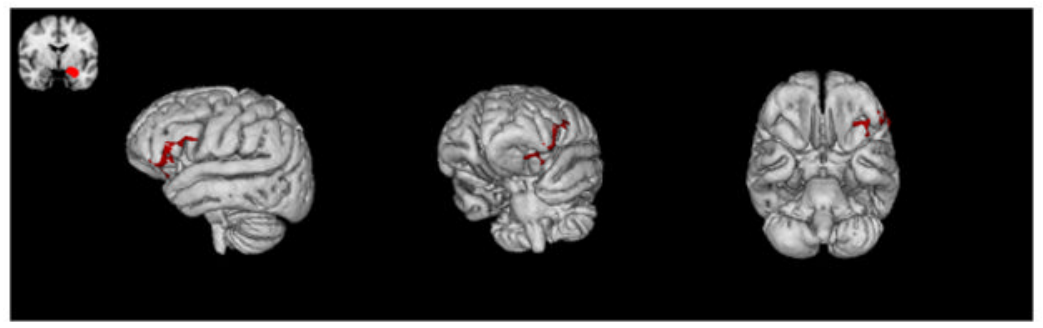

Figure 5. Amygdala-Prefrontal Functional Connectivity

CHR patients demonstrated weaker inverse task-dependent functional connectivity between the amygdala and prefrontal regions consisting of left orbitofrontal cortex and left inferior frontal gyrus, compared with controls $(\mathrm{Z}=3.82, \mathrm{p}=.027$, corrected $)$. 
Table 1

Demographic and Clinical Characteristics

\begin{tabular}{|c|c|c|}
\hline & $\underset{n=14}{\text { Controls }}$ & $\begin{array}{c}\text { Patients } \\
\mathbf{n}=\mathbf{2 0}\end{array}$ \\
\hline Age (years, SD) & $18.7(2.5)$ & $18.8(2.4)$ \\
\hline Sex (male/female) & $5 / 9$ & $10 / 10$ \\
\hline \multicolumn{3}{|l|}{ Primary prodromal criteria (n) } \\
\hline Attenuated Positive Symptoms (APS) ${ }^{*}$ & $\mathrm{n} / \mathrm{a}$ & 17 \\
\hline Brief Intermittent Psychotic Symptoms (BIPS) & $\mathrm{n} / \mathrm{a}$ & 1 \\
\hline Youth \& Schizotypy (YS) & $\mathrm{n} / \mathrm{a}$ & 2 \\
\hline GAF score (mean, SD) & $88.8(5.4)$ & $50.3(9.4)$ \\
\hline \multicolumn{3}{|l|}{ Race/ethnicity (n) } \\
\hline White (European) & 5 & 10 \\
\hline Hispanic & 3 & 3 \\
\hline Black & 1 & 2 \\
\hline Southeast Asian & 1 & 2 \\
\hline Interracial & 3 & 1 \\
\hline First Nations (North American Indian, Metis, Inuit) & 0 & 1 \\
\hline Native Hawaiian or Pacific Islander & 0 & 1 \\
\hline West/Central Asian or Middle Eastern & 1 & 0 \\
\hline \multicolumn{3}{|l|}{ Medication status (n) } \\
\hline Current atypical antipsychotic & 0 & 5 \\
\hline Current antidepressant & 0 & 6 \\
\hline Current psychostimulant & 0 & 2 \\
\hline Current other (Abilify, Cogentin, Klonopin) & 0 & 3 \\
\hline No current medication & 14 & 12 \\
\hline One current medication & 0 & 2 \\
\hline Two current medications & 0 & 3 \\
\hline Three current medications & 0 & 2 \\
\hline Four current medications & 0 & 1 \\
\hline
\end{tabular}

* Two CHR participants with Attenuated Positive Symptoms (APS) also met criteria for Youth \& Schizotypy (YS), and one other CHR participant with APS also met criteria for Genetic Risk \& Deterioration (GRD).

Demographic information and clinical characteristics are displayed for the sample. There was no significant difference in the age or gender distribution of the groups. For the CHR patient group, the majority of participants met Attenuated Positive Symptom (APS) criteria. Brief Intermittent Psychotic Symptom (BIPS), Genetic Risk and Deterioration (GRD), and Youth and Schizotypy (YS) were also considered inclusion criteria for the prodromal group. CHR patients displayed significantly poorer functioning compared with controls, as measured by GAF score ( $\mathrm{p}<$. 001). 


\section{Table 2}

Mean Accuracy (\%) by Group

\begin{tabular}{lcccc}
\hline & Patients & Controls & $\mathbf{t}$ & $\mathbf{p}$ \\
Total & 92.6 & 93.2 & -0.270 & 0.789 \\
Shape Match & 92.5 & 95.4 & -0.877 & 0.387 \\
Emotion Match & 90.8 & 88.9 & 0.442 & 0.661 \\
Gender Match & 94.5 & 90.7 & 0.853 & 0.400 \\
Gender Label & 93.5 & 97.5 & -1.534 & 0.135 \\
Emotion Label & 92.0 & 93.6 & -0.697 & 0.491 \\
\hline
\end{tabular}

Mean accuracy scores (in percentage correct) are displayed for patients and controls. There were no significant differences in accuracy of performance between groups. 


\section{Table 3}

Mean Reaction Time (msec) by Group

\begin{tabular}{lcccc}
\hline & Patients & Controls & t & $\mathbf{p}$ \\
Total & 1522 & 1537 & -0.154 & 0.878 \\
Shape Match & 1196 & 1244 & -0.518 & 0.608 \\
Emotion Match & 1936 & 1929 & 0.062 & 0.951 \\
Gender Match & 1294 & 1354 & -0.562 & 0.578 \\
Gender Label & 1459 & 1442 & 0.172 & 0.865 \\
Emotion Label & 1747 & 1715 & 0.245 & 0.808 \\
\hline
\end{tabular}

Mean reaction times (in $\mathrm{msec}$ ) are shown for patients and controls. There were no significant differences in reaction time between groups. 


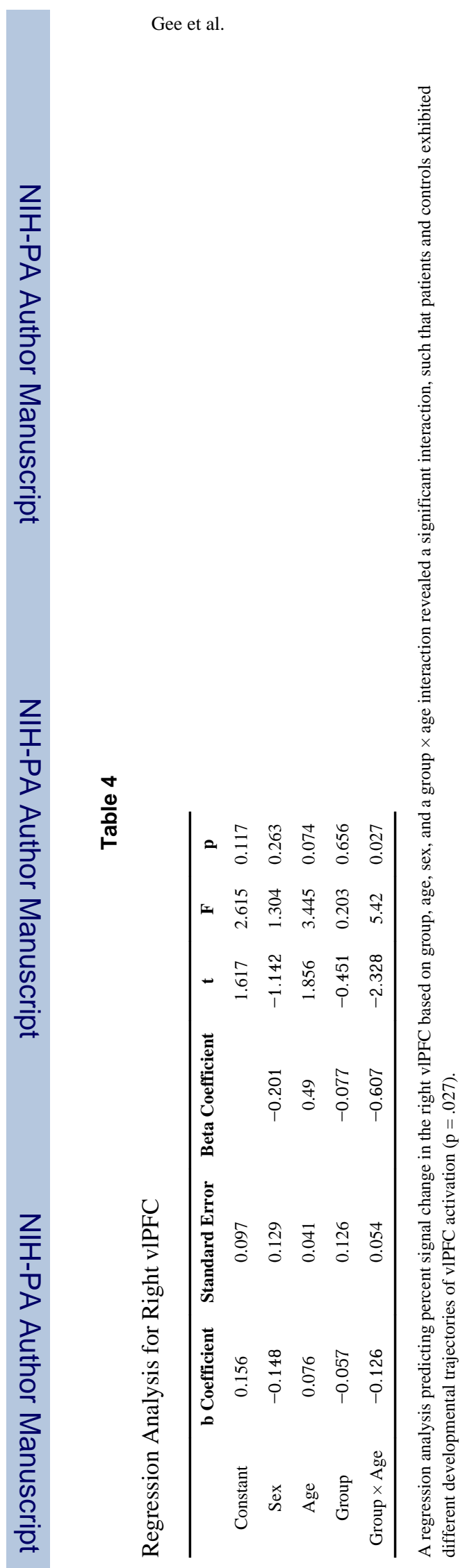

Schizophr Res. Author manuscript; available in PMC 2013 January 1. 


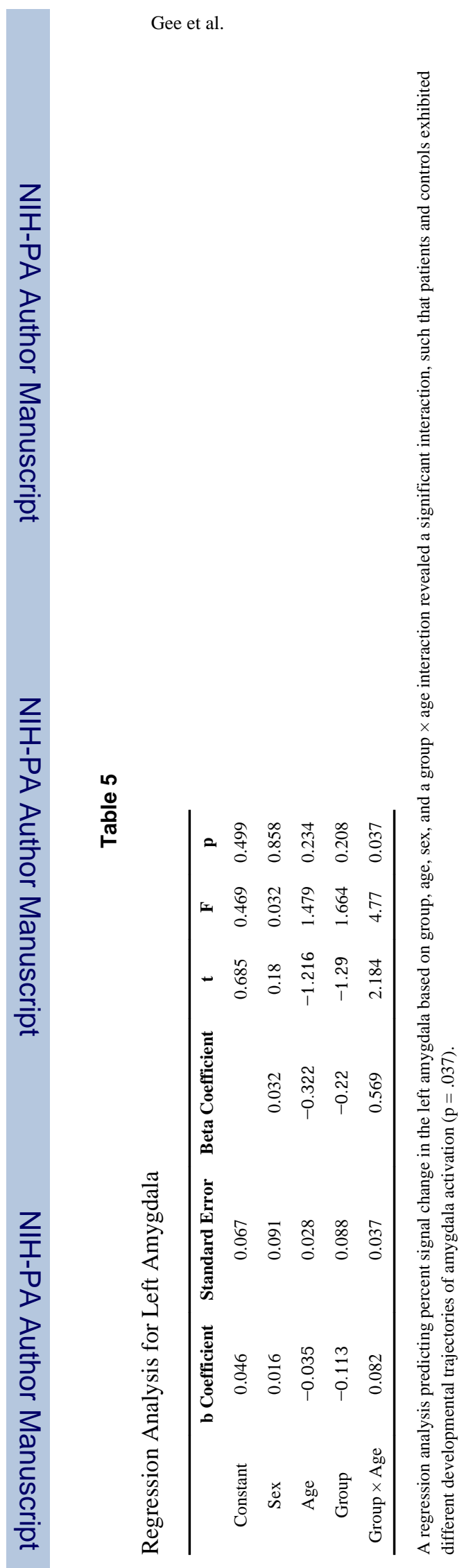

Schizophr Res. Author manuscript; available in PMC 2013 January 1. 